\title{
OPEN Statistical modeling for bioconvective tangent hyperbolic nanofluid towards stretching surface with zero mass flux condition
}

\author{
Anum Shafiq ${ }^{1}$, S. A. Lone ${ }^{2}$, Tabassum Naz Sindhu ${ }^{3}$, Q. M. Al-Mdallal ${ }^{4 \bowtie} \&$ G. Rasool ${ }^{5}$
}

This article presents the implementation of a numerical solution of bioconvective nanofluid flow. The boundary layer flow (BLF) towards a vertical exponentially stretching plate with combination of heat and mass transfer rate in tangent hyperbolic nanofluid containing microorganisms. We have introduced zero mass flux condition to achieve physically realistic outcomes. Analysis is conducted with magnetic field phenomenon. By using similarity variables, the partial differential equation which governs the said model was converted into a nonlinear ordinary differential equation, and numerical results are achieved by applying the shooting technique. The paper describes and addresses all numerical outcomes, such as for the Skin friction coefficients (SFC), local density of motile microorganisams (LDMM) and the local number Nusselt (LNN). Furthermore, the effects of the buoyancy force number, bioconvection Lewis parameter, bioconvection Rayleigh number, bioconvection Pecelt parameter, thermophoresis and Brownian motion are discussed. The outcomes of the study ensure that the stretched surface has a unique solution: as $\mathrm{Nr}(\mathrm{Lb})$ and $R b(P e)$ increase, the drag force (mass transfer rate) increases respectively. Furthermore, for least values of $N b$ and all the values of $\mathrm{Nt}$ under consideration the rate of heat transfer upsurges. The data of SFC, LNN, and LDMM have been tested utilizing various statistical models, and it is noted that data sets for SFC and LDMM fit the Weibull model for different values of $N r$ and $L b$ respectively. On the other hand, Frechet distribution fits well for LNN data set for various values of $N t$.

Recently, several studies have been conducted on stretching surfaces, that are used in industrial materials like glass fibers and lubricants. Crane ${ }^{1}$ suggested the flowing mechanisms towards a stretched surface. Investigators ${ }^{2}$ have studied heat transfer phenomenon using permeable stretching sheet. Numerous other researchers performed similar studies involving a stretching surface $\left(\mathrm{se}^{3-8}\right)$. Convective heat transfer is a significant feature of nanofluids, and it has been found that incorporating nanomaterials enhances the thermal conductivity. Nanofluids have received extensive interest of recent investigators because of their numerous potential usages like in power generation, nuclear reactors, electronics, biomedicine, chemical processes, space technology and nanotechnology. $\mathrm{In}^{9}$, Makinde and Aziz analyzed boundary layer (BL) stream of nanoliquid towards a stretched plate via CBC (convective boundary conditions). $\mathrm{In}^{10}$, combined impacts of heat, mass phenomena in stream of nanoliquids towards a non-horizontal surface via radiation is scrutinized. In ${ }^{11}$, Mustafa et al. investigated unsteady BL flow of nanoliquid towards a stretching surface. $\operatorname{In}^{12}$, Ashorynejad et al. analyzed heat transfer characteristics of nanoliquid by incorporating MHD effect. Murthy et al. ${ }^{13}$ examined convection heat transfer phenomenon in stratified nanoliquid under non-Darcy porous phenomenon. The formulation of entropy generation using nanoliquid via rotating porous plate was reported by Rashidi et al. ${ }^{14}$. Jedi et al. ${ }^{15}$ studied statistical modeling of nanofluid flow towards the stretching surface. They gave the concept of modeling the data of considered studied statistically

\footnotetext{
${ }^{1}$ School of Mathematics and Statistics, Nanjing University of Information Science and Technology, Nanjing 210044, China. ${ }^{2}$ Department of Basic Science, College of Science and Theoretical Studies, Saudi Electronic University, Riyadh, Kingdom of Saudi Arabia. ${ }^{3}$ Department of Statistics, Quaid-i-Azam University 4250, Islamabad 44000, Pakistan. ${ }^{4}$ Department of Mathematical Sciences, UAE University, P.O. Box 15551 Al-Ain, United Arab Emirates. ${ }^{5}$ Binjiang College, Nanjing University of Information Science and Technology, Wuxi 214105, Jiangsu, China. ${ }^{\boxplus}$ email: q.almdallal@uaeu.ac.ae
} 


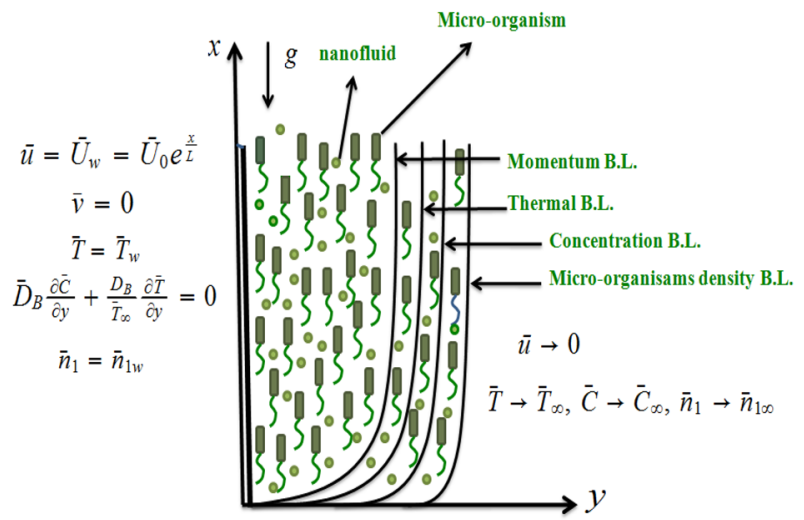

Figure 1. Physical model.

via incorporating statistical distributions. Chu et al. ${ }^{16,17}$ studied ANN modeling of nanofluid examined experimentally and then the results were compared with regression-based methodologies.

Bioconvection has various uses in biological and biotechnological processes. The bioconvection term indicates a macroscopic convective movement of liquid induced by density gradient produced due to joint swimming of motile microorganisms. By moving in a specific direction, such self-propelled motile microorganisms rise density of base liquid, thereby initiating bioconvection. The bioconvection process in nanofluid convection is associated with presence of denser microorganisms that accumulate on lighter water surface. As heavier microbes fall into water, up-swimming microbes replenish them, thus creating the mechanism of bioconvection within system. The mechanism is a mesoscale phenomenon where a macroscopic movement is caused by motion of motile micro-organisms (MMs). Nanomaterials are not self-propelled unlike motile microorganisms. Their movement is driven by thermophoresis and Brownian phenomena happening in nanofluid. Therefore, movement of MMs (motile micro-organisms) is free of movement of nanoparticles. The addition of micro-organisms to a nanofluid improves its stability as a suspension ${ }^{18}$ and may prevent aggregation and agglomeration of nanoparticles. $\operatorname{In}^{19}$, Aziz et al. studied free convective BL flow over a horizontal surface in nanoliquid including gyrotactic microorganisms. They noted that bioconvective numbers significantly influenced mass, motile micro-organism and heat transfer rate. $\mathrm{In}^{20}$, Tham et al. numerically examined mixed convective BL flow about a solid surface saturated in porous medium via nanoliquid including gyrotactic microorganisms by considering heated and cooling sphere. $\mathrm{In}^{21}$ Ibrahim studied the time-dependent viscous fluid flow due to a rotating stretchable disk.

A comprehensive explanation ${ }^{22-34}$ is given for onset of bioconvection in suspension of oxytactic/gyrotactic micro-organisms in different situations.

Motivated by Jedi et al. ${ }^{15}$, we have investigated the BLF of tangent hyberbolic nanoliquid containing gyrotactic microorganisms with zero mass flux condition. Our main aim here is to find effect of key numbers (buoyancy force parameter, bioconvection Rayleigh parameter, thermophoresis, Brownian motion, bioconvective Lewis number and bioconvective Pecelt number). The shooting methodology along with RK4 has utilized to gain the outcomes for SFC, LNN and LDMM. In order to estimate thermal conductivity of a nanoliquid containing microorganisms, a physical-statistical model, as well as its distribution is considered. In further research on nanoliquids containing microorganisms, the proposed model could be used for a wide variety of practical uses.

\section{Formulation}

The steady BL flow of tangent hyperbolic nanoliquid containing microorganisams over a vertically exponential stretching plate with zero mass flux condition is considered. The MHD and Joule heating phenomena in the absence of viscous dissipation is considered into account. The physical configuration scheme is illustrated in Fig. 1. The current flow is driven by following set of equations ${ }^{26,27}$ :

$$
\begin{gathered}
\frac{\partial \bar{u}}{\partial x}+\frac{\partial \bar{v}}{\partial y}=0 \\
\bar{u} \frac{\partial \bar{u}}{\partial x}+\bar{v} \frac{\partial \bar{u}}{\partial y}=v(1-n) \frac{\partial^{2} \bar{u}}{\partial y^{2}}+2 v \Gamma n \frac{\partial \bar{u}}{\partial y} \frac{\partial^{2} \bar{u}}{\partial y^{2}}-\frac{\sigma}{\bar{\rho}} B_{0}^{2} \bar{u}+\frac{1}{\bar{\rho}}\left[\left(1-\bar{C}_{\infty}\right) \bar{\rho} \beta_{T} g\left(\bar{T}-\bar{T}_{\infty}\right)\right. \\
\left.-\left(\bar{\rho}_{p}-\bar{\rho}\right) g\left(C-C_{\infty}\right)-\left(\bar{n}_{1}-\bar{n}_{1 \infty}\right) g \gamma\left(\bar{\rho}_{m}-\bar{\rho}\right)\right] \\
u \frac{\partial \bar{T}}{\partial x}+v \frac{\partial \bar{T}}{\partial y}=\frac{\kappa}{\rho c_{p}} \frac{\partial^{2} \bar{T}}{\partial y^{2}}+\bar{\tau}\left[D_{B} \frac{\partial \bar{T}}{\partial y} \frac{\partial C}{\partial y}+\frac{D_{T}}{T_{\infty}}\left(\frac{\partial \bar{T}}{\partial y}\right)^{2}\right]+\frac{\sigma}{\rho c_{p}} \bar{B}_{0}^{2} \bar{u}^{2},
\end{gathered}
$$




$$
\begin{gathered}
\bar{u} \frac{\partial C}{\partial x}+\bar{v} \frac{\partial C}{\partial y}=\frac{D_{T}}{T_{\infty}} \frac{\partial^{2} \bar{T}}{\partial y^{2}}+D_{B} \frac{\partial^{2} C}{\partial y^{2}} \\
\bar{u} \frac{\partial n_{1}}{\partial x}+\bar{v} \frac{\partial n_{1}}{\partial y}+\frac{\bar{b} W_{c}}{\left(C_{w}-C_{\infty}\right)} \frac{\partial}{\partial y}\left(n_{1} \frac{\partial C}{\partial y}\right)=D_{m} \frac{\partial^{2} n_{1}}{\partial y^{2}},
\end{gathered}
$$

with

$$
\begin{aligned}
& \bar{u}=\bar{U}_{w}=\bar{U}_{0} e^{\frac{x}{L}}, \bar{v}=0, \bar{T}=\bar{T}_{w}, D_{B} \frac{\partial C}{\partial y}+\frac{D_{B}}{\bar{T}_{\infty}} \frac{\partial \bar{T}}{\partial y}=0, n_{1}=n_{1 w} \text { at } y=0, \\
& \bar{u} \rightarrow 0, \bar{T} \rightarrow \bar{T}_{\infty}, C \rightarrow C_{\infty}, n_{1} \rightarrow n_{1 \infty} \text { when } y \rightarrow \infty .
\end{aligned}
$$

Here velocity components $(\bar{u}, \bar{v})$ in $(x, y)$ directions respectively, $\bar{\rho}$ density of nanoliquid, $\mu$ viscosity of nanoliquid and microorganisms, density of nanomaterials is $\bar{\rho}_{p}$, electrical conductivity of nanoliquid is $\sigma$, density of microorganisms materials $\bar{\rho}_{m}$, heat capacity ratio of nanomaterials by nanoliquid is $\bar{\tau}=\frac{(\bar{\rho} c)_{p}}{(\bar{\rho} c)_{f}}$, temperature of liquid is $\bar{T}$, density motile of microorganisms is $n_{1}$, concentration of nanomaterials $C$, kinematic viscosity $v$, volume expansion coefficient of liquid $\beta_{T}$, gravity is $g$, average volume of a micro-organism $\gamma$, specific heat $c_{p}, \bar{U}_{w}$ is the stretching velocity, chemotaxis constant $\bar{b}$ and maximum cell swimming speed $W_{c}$, thermophoretic diffusion coefficient $D_{T}$, Brownian motion diffusion coefficient $D_{B}$, ambient temperature $\bar{T}_{\infty}$, ambient concentration of nanoparticles $C_{\infty}$, ambient microorganisms concentration $n_{1 \infty}$. and $D_{m}$ is diffusivity of microorganisms.

Using the below mentioned transformations

$$
\begin{aligned}
& \bar{u}=\bar{U}_{0} e^{\frac{x}{L}} f^{\prime}(\eta), \bar{v}=-\left(\frac{v \bar{U}_{0}}{2 L}\right)^{\frac{1}{2}} e^{\frac{x}{2 L}}\left(f+\eta f^{\prime}\right), \eta=\left(\frac{\bar{U}_{0}}{2 v L}\right)^{\frac{1}{2}} e^{\frac{x}{2 L}} y, \\
& \theta=\frac{\bar{T}-\bar{T}_{\infty}}{\bar{T}_{w}-\bar{T}_{\infty}}, \phi=\frac{C-C_{\infty}}{C_{w}-C_{\infty}}, \xi=\frac{n_{1}-n_{1 \infty}}{n_{1 w}-n_{1 \infty}},
\end{aligned}
$$

the continuity equation is identically satisfied and Eqs. (2-6) becomes

$$
\begin{gathered}
(1-n) f^{\prime \prime \prime}-2\left(f^{\prime}\right)^{2}+f^{\prime \prime}+n W e f^{\prime \prime} f^{\prime \prime \prime}-M^{2} f^{\prime}+\lambda[\theta-N r \phi-R b \xi]=0, \\
\theta^{\prime \prime}+\operatorname{Pr} f \theta^{\prime}+\operatorname{Pr} N t\left(\theta^{\prime}\right)^{2}+\operatorname{Pr} N b \phi^{\prime} \theta^{\prime}+\operatorname{Pr} M^{2} E c\left(f^{\prime}\right)^{2}=0, \\
\phi^{\prime \prime}+\operatorname{Lef} \phi^{\prime}+\frac{N t}{N b} \theta^{\prime \prime}=0, \\
\left.\xi^{\prime \prime}+\operatorname{Lbf} \xi^{\prime}-\operatorname{Pe}(\xi+1) \phi^{\prime \prime}-\operatorname{Pe} \xi^{\prime} \phi^{\prime}\right]=0, \\
f(0)=0, f^{\prime}(0)=1, \theta(0)=1, \quad N b \phi^{\prime}(0)+N t \theta^{\prime}(0)=0, \quad \xi(0)=1, \\
f^{\prime}(\infty) \rightarrow 0, \quad \theta(\infty) \rightarrow 0, \quad \phi(\infty) \rightarrow 0, \quad \xi(\infty) \rightarrow 0 .
\end{gathered}
$$

The dimensionless parameters are

$$
\begin{aligned}
\lambda & =\frac{2 L g \beta_{T}\left(1-\bar{C}_{\infty}\right) \bar{\rho}_{f}\left(\bar{T}_{w}-\bar{T}_{\infty}\right)}{U_{0}^{2}}, M^{2}=\frac{2 L \sigma B_{0}^{2} e^{-\frac{x}{L}}}{\bar{\rho}_{f} U_{0}}, W e=\frac{\sqrt{2} \Gamma U_{0}^{\frac{3}{2}} e^{\frac{3 x}{2 L}}}{\sqrt{v L}}, \\
N r & =\frac{\left(\bar{C}_{w}-\bar{C}_{\infty}\right)\left(\bar{\rho}_{p}-\bar{\rho}_{f}\right)}{\beta \rho_{f}\left(1-\bar{C}_{\infty}\right) \bar{T}_{\infty}}, R_{b}=\frac{\left(\bar{\rho}_{m}-\bar{\rho}_{f}\right) \gamma\left(\bar{n}_{1 w}-\bar{n}_{1 \infty}\right)}{\bar{\rho}_{f}\left(1-\bar{C}_{\infty}\right) \beta\left(\bar{T}_{w}-\bar{T}_{\infty}\right)}, N t=\frac{(\bar{\rho} c)_{p} \bar{D}_{T}\left(\bar{T}_{w}-\bar{T}_{\infty}\right)}{v(\bar{\rho} c)_{f}}, \\
N b & =\frac{(\bar{\rho} c)_{p} \bar{D}_{B}\left(\bar{C}_{w}-\bar{C}_{\infty}\right)}{v(\bar{\rho} c)_{f}}, L e=\frac{v}{\bar{D}_{B}}, L b=\frac{v}{\bar{D}_{m}}, E c=\frac{U_{0}^{2} e^{\frac{2 x}{L}}}{c_{p} k\left(\bar{T}_{w}-\bar{T}_{\infty}\right)}, \\
P_{e} & =\frac{\bar{b} W_{c}}{\bar{D}_{m}}, \quad \operatorname{Pr}=\frac{\mu c_{p}}{\kappa} .
\end{aligned}
$$

in which $\lambda$ represents mixed convective parameter, $M$ represents magnetic number, We represents Weissenberg number, $\mathrm{Nr}$ represents buoyancy force number, $N b$ represents Brownian motion parameter, $R_{b}$ represents bioconvection Rayleigh number, $L b$ represents bioconvection Lewis number, $N t$ represents thermophoresis parameter, $L e$ represents Lewis parameter, $E c$ represents Eckert parameter, $P_{e}$ represents bioconvective Pecelt parameter and Pr represents Prandtl parameter.

Dimensional SFC, LNN and LDMM become 


$$
\begin{gathered}
C_{f x}=\frac{2 \tau_{x y}}{\rho U_{w}^{2}}, \quad N_{u x}=\frac{x q_{w}}{K\left(T-T_{\infty}\right)}, N_{n x}=\frac{x q_{n}}{D_{n}\left(n-n_{\infty}\right)}, \\
\tau_{x y}=\mu\left((1-n) \frac{\partial \bar{u}}{\partial y}+2 \Gamma n\left(\frac{\partial \bar{u}}{\partial y}\right)^{2}\right), \quad q_{w}=\left.\frac{\partial T}{\partial y}\right|_{y=0}, \quad q_{n}=\left.\frac{\partial n_{1}}{\partial y}\right|_{y=0} .
\end{gathered}
$$

The dimensionless form of SFC, LNN and LDMM are

$$
\begin{gathered}
\left(\frac{\operatorname{Re}}{2}\right)^{1 / 2} C_{f x}=(1-n) f^{\prime \prime}(0)+\frac{n}{2} W e\left(f^{\prime \prime}(0)\right)^{2} . \\
\left(\frac{\operatorname{Re}_{x}}{2}\right)^{-1 / 2} N_{u x}=-\theta^{\prime}(0), \\
\left(\frac{\operatorname{Re}_{x}}{2}\right)^{-1 / 2} N_{n x}=\xi^{\prime}(0),
\end{gathered}
$$

where $\operatorname{Re}_{x}=\frac{U_{0} L e^{\frac{x}{L}}}{v}$ is the local Reynold number.

\section{Model selection: AIC and BIC}

Model selection process are guidelines that are used to choose a statistical model from a list of candidates depending on data. The first broad metric for selecting models estimated by maximum likelihood was proposed by Akaike ${ }^{35}$. The AIC is the most commonly used model selection method in statistics. One can determine the best fit for the data by calculating and comparing the AIC scores of various different models. Using the maximum likelihood estimate and the number of parameters in the model, AIC calculates the relative information value of the model. This criterion, is widely regarded as the first model selection criterion to be employed in practise. The Bayesian Information Criterion, or BIC for short, is another prominent model selection criterion. Bayesian probability and inference is the subject of research from which it was derived. It's appropriate for models that fit within the maximum likelihood estimation framework, just like AIC. Other prominent model selection methods include the AIC corrected for small-sample bias (AICc) and the Hannan-Quinn criterion (HQC). The data for the SFC, LNN and LDMM were tested using Akaike information criterion (AIC) and the Bayesian Information Criterion (BIC). These test are utilized to determine goodness of fit and find the model that fits best to data. The different statistical models are mentioned in Table 1. The AIC and BIC were determined for each model in the table, and the best distribution was identified from the values of AIC and BIC. The AIC/BIC determines the quality of statistical distributions for a sample set of data. The model which gives the lowest BIC/AIC value best fits the data. The formula for the AIC and BIC are

$$
A I C=-2 \log (L)+2 k,
$$

where $k$ is the number of estimated parameters and $L$ is the maximized likelihood function in the model.

$$
B I C=-2 \log (L)+k \log (n) .
$$

\section{Discussion}

The shooting methodology was utilized to achieve the numerical simulation for (8)-(11) with boundary conditions (12). The shooting techniques transform a BVP (boundary value problem) into an IVP (initial value problem). This methodology was employed by using "dsolve" command and the "shoot" implementation in Mathematica programming language. The influences of $N r, N t$, and $L b$ on the SFC, LNN and LDMM were investigated.

Figure $2 \mathrm{a}-\mathrm{c}$ depicts the variation in the SFC, LNN and LDMM for different significant physical parameters. It is observed from Fig. 2a that as Nr increases, SFC increases, while Fig. 2b clearly shown that LNN is increasing function of $N t$ when $N b$ ranges from 0 to 15 . Figure $2 \mathrm{c}$ is plotted for the various values of Lb for LDMM when $0 \leq P_{e} \leq 15$. Figure $2 \mathrm{c}$ shows the same trend for $L b$. It is worth remembering that the Brownian motion parameter $\mathrm{Nb}$ and the thermophoresis $\mathrm{Nt}$ are associated with the nanoparticles' random motion. For smaller values of $\mathrm{Nb}$ and $\mathrm{Nt}$, the fluid viscosity is low, and the nanomaterials and microorganisms tend to pass easily between each other. The fluid is cooled faster because of this phenomenon, and heat transfer rate increases. The contour plot is sketched for the same parameters corresponds to SFC, LNN and LDMM (see Fig. 3a-c).

The data for the skin friction coefficient, Nusselt and density of motile microorganisam numbers were further analysed on the basis of Fig. 2, in order to obtain the statistical properties for the tested models. Tables 2, 3 and 4 present the estimated parameters of the different distributions that have been tested with the considered data. Tables 5, 6 and 7 demonstrate the Akaike Information Criteria (AIC) and Bayesian Information Criterion (BIC) for the SFC, LNN and LDMM numbers.

By using AIC and BIC as the model selection criteria, it is noticed that the Weibull distribution is suitable for modelling the SFC and LDMM (see Tables 5, 7). On the other side, for LNN, Frechet distribution is suitable under both AIC and BIC criteria. The estimated densities using data of SFC, LNN and LDMM under abovementioned models (see Figs. 4, 5, 6). Through these figures it can be observed that the Weibull distribution is best fitted model for SFC and LDMM. While, Frechet distribution is best fitted for LNN. 


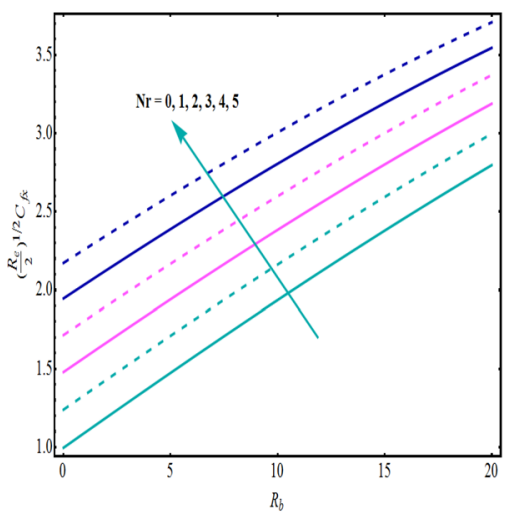

(a)

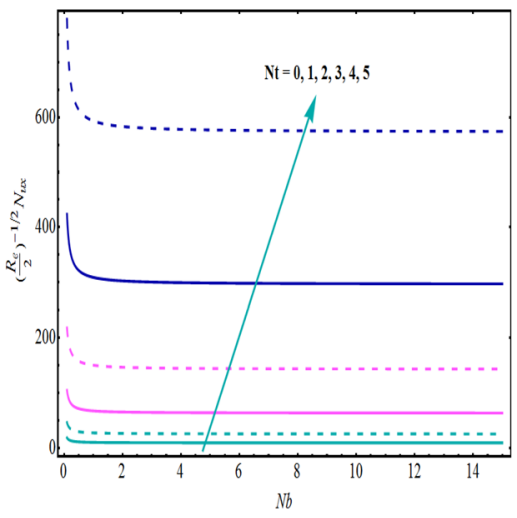

(b)

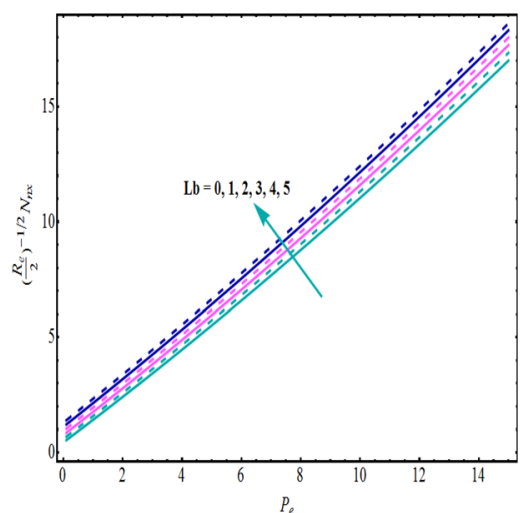

(c)

Figure 2. Variations for (a) SFC, (b) LNN, (c) LDMM with $R_{b}, N b, P_{e}$ with various values of $N r, N t$ and $P_{e}$, respectively.

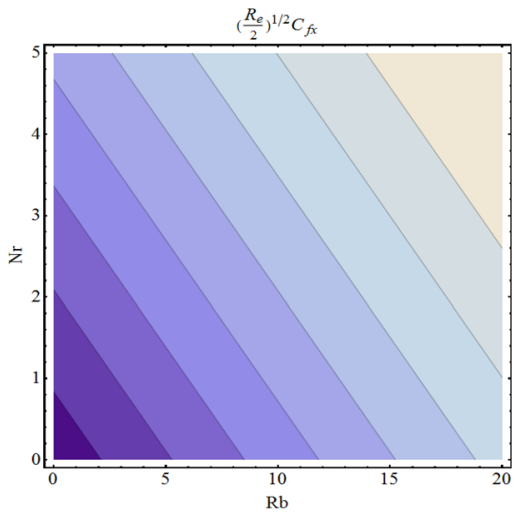

(a)

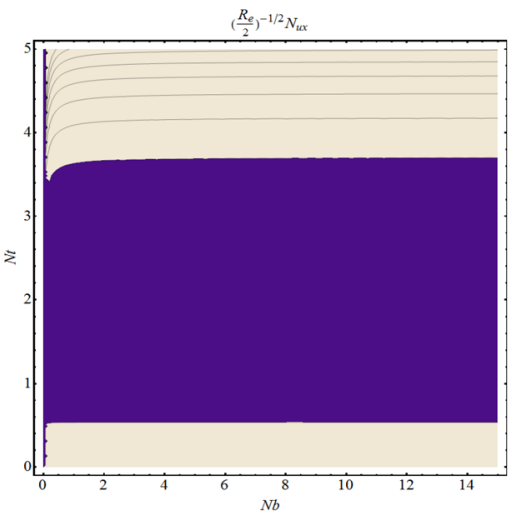

(b)

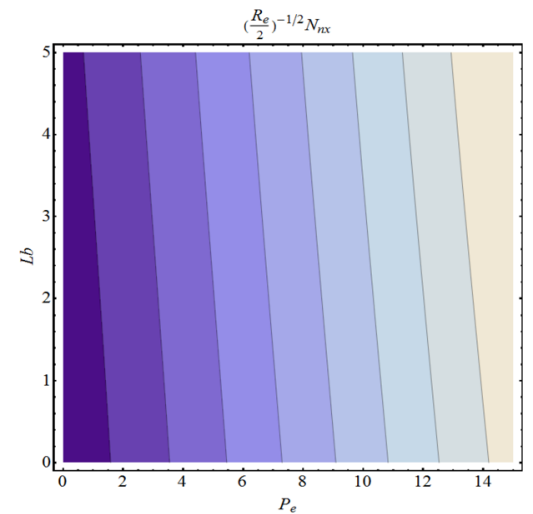

(c)

Figure 3. Contour graphs for (a) SFC, (b) LNN, (c) LDMM with $R_{b}, N b, P_{e}$ with various values of $N r, N t$ and $P_{e}$, respectively.

\begin{tabular}{|l|l|}
\hline Distribution & Probability distribution function $f(x)$ \\
\hline Frechet distribution & $f(x ; \alpha, \beta)=\frac{\beta}{x}\left(\frac{\alpha}{x}\right)^{\beta} e^{-\left(\frac{\alpha}{x}\right)^{\beta}} x>0, \alpha, \beta>0$, \\
\hline & where $\alpha, \beta$ are shape parameters. \\
\hline Additive Gumbel Type II Distribution & $f\left(x ; \eta_{i}, \delta_{i}\right)=\sum_{i=1}^{2} \eta_{i} \delta_{i} x^{-\eta_{i}-1} e^{-\sum_{i=1}^{2} \delta_{i} x^{-\eta_{i}} x,\left(\delta_{i}, \eta_{i}\right)>0}$ \\
\hline Gumbel Type II Distribution & Where the shape parameters are $\delta_{i}, \eta_{i}, i=1,2$. \\
\hline & $f(x ; \eta, \delta)=\eta \delta x^{-\eta-1} e^{-\delta x^{-\eta}}(x, \delta, \eta)>0$, \\
\hline Weibull Distribution & Where the shape parameters are $\delta, \eta$. \\
\hline & $f(x ; \lambda, \beta)=\frac{\beta}{\lambda}\left(\frac{x}{\lambda}\right)^{\beta-1} e^{-\left(\frac{x}{\lambda}\right)^{\beta}} x>0, \lambda, \beta>0$, \\
\hline & where $\beta>0$ is the shape parameter and $\lambda>0$ \\
\hline Modified Frechet distribution & is the scale parameter of the distribution. \\
\hline & $f(x ; \alpha, \beta, \lambda)=\frac{1}{x}(\beta+\lambda x)\left(\frac{\alpha}{x}\right)^{\beta}$ exp $\left[-\lambda x-\left(\frac{\alpha}{x}\right)^{\beta}\right]$ \\
\hline & $x>0, \alpha, \beta>0, \lambda \geq 0$, \\
\hline Rayleigh Distribution & where $\alpha, \beta, \lambda$ are shape parameters. \\
\hline & $f(x ; \sigma)=\frac{x}{\sigma^{2}}$ exp $\left[-\frac{x^{2}}{2 \sigma^{2}}\right]$ \\
\hline & where $\sigma$ is the scale parameter of the distribution. \\
\hline
\end{tabular}

Table 1. The distribution test for SFC, LNN and LDMM. 


\begin{tabular}{|c|c|c|c|c|c|c|c|}
\hline & & \multicolumn{6}{|l|}{ SFC } \\
\hline & & \multicolumn{6}{|l|}{$\mathrm{Nr}$} \\
\hline & & 0 & 1 & 2 & 3 & 4 & 5 \\
\hline \multirow{3}{*}{$\mathrm{FD}$} & $\hat{\alpha}$ & 1.568950 & 1.812761 & 2.048765 & 2.277253 & 2.498126 & 2.711093 \\
\hline & $\hat{\beta}$ & 3.249076 & 3.819011 & 4.407746 & 5.023453 & 5.674475 & 6.370279 \\
\hline & $\hat{\eta}_{1}$ & 2.340444 & 9.769603 & 14.49602 & 61.68615 & -3.898806 & 8.543967 \\
\hline \multirow{3}{*}{ AGT-II } & $\hat{\delta}_{1}$ & 1.980203 & 2.195986 & 9.106387 & 38.38976 & 173.47197 & 565.10015 \\
\hline & $\hat{\eta}_{2}$ & 3.249075 & 3.820303 & 4.407449 & 5.008879 & 37.15317 & 23.634421 \\
\hline & $\hat{\delta}_{2}$ & 3.249069 & 14.637496 & 4.408063 & 32.58794 & 5.630879 & 6.353374 \\
\hline \multirow{2}{*}{ GT-II } & $\hat{\eta}$ & 3.249075 & 3.818910 & 4.404736 & 4.949803 & 4.720524 & 4.381971 \\
\hline & $\hat{\delta}$ & 4.320648 & 9.696103 & 23.54969 & 58.52508 & 73.90224 & 75.942714 \\
\hline \multirow{3}{*}{ Weibull } & $\hat{\lambda}$ & 4.038674 & 4.644257 & 5.278253 & 5.950158 & 6.670540 & 7.452207 \\
\hline & $\hat{\beta}$ & 2.126016 & 2.354407 & 2.574283 & 2.785597 & 2.988088 & 3.181357 \\
\hline & $\hat{\alpha}$ & 27.63889 & 0.0636608 & 0.1125029 & 0.182326 & 0.2705209 & 0.3647409 \\
\hline \multirow{2}{*}{ MFD } & $\hat{\beta}$ & 0.847507 & -1.6221723 & -2.2936863 & -3.249356 & -4.5623501 & -6.2105315 \\
\hline & $\hat{\lambda}$ & 1.480263 & 2.8919409 & 3.1625153 & 3.528906 & 3.9943797 & 4.5362588 \\
\hline $\mathrm{RD}$ & $\hat{\sigma}$ & 1.412951 & 1.564143 & 1.712606 & 1.857528 & 1.998237 & 2.134108 \\
\hline
\end{tabular}

Table 2. Estimates of the parameters of statistical distribution for SFC.

\begin{tabular}{|c|c|c|c|c|c|c|c|}
\hline & & \multicolumn{6}{|l|}{ LNN } \\
\hline & & \multicolumn{6}{|l|}{$N t$} \\
\hline & & 6 & 7 & 8 & 9 & 10 & 11 \\
\hline \multirow{3}{*}{ FD } & $\hat{\alpha}$ & 9.140826 & \begin{tabular}{|l|}
25.54203 \\
\end{tabular} & \begin{tabular}{|l|}
63.89860 \\
\end{tabular} & 144.3362 & \begin{tabular}{|l|}
299.1832 \\
\end{tabular} & \begin{tabular}{|l|}
577.6288 \\
\end{tabular} \\
\hline & $\hat{\beta}$ & 19.18480 & 22.74168 & 27.43727 & 32.98885 & 39.12873 & 45.97684 \\
\hline & $\hat{\eta}_{1}$ & 78.15356 & 99.97835 & 144.78854 & 213.8291 & 135.2225 & \begin{tabular}{|l|l|}
9.497816 \\
\end{tabular} \\
\hline \multirow{3}{*}{ AGT-II } & $\hat{\delta}_{1}$ & 97.92131 & 84.87295 & 137.58036 & 206.7033 & 125.0395 & 2.050675 \\
\hline & $\hat{\eta}_{2}$ & 2.394169 & 1.733487 & 1.373428 & 1.243163 & \begin{tabular}{|l|l|}
0.987689 \\
\end{tabular} & 3.005450 \\
\hline & $\hat{\delta}_{2}$ & 2.398008 & 1.618335 & 1.434913 & 1.247597 & 0.993976 & 1.217642 \\
\hline \multirow{2}{*}{ GT-II } & $\hat{\eta}$ & 2.171243 & 1.453281 & 1.202308 & 0.972356 & \begin{tabular}{|l|l|}
0.827369 \\
\end{tabular} & \begin{tabular}{|l|l|}
0.740383 \\
\end{tabular} \\
\hline & $\hat{\delta}$ & 101.4447 & 94.73049 & 125.691247 & 106.4941 & 87.12970 & 85.61986 \\
\hline \multirow{3}{*}{ Weibull } & $\hat{\lambda}$ & 3.618602 & 4.251510 & 5.089174 & 6.074484 & 7.143974 & 1.756068 \\
\hline & $\hat{\beta}$ & 10.66843 & 29.12578 & 71.293623 & 158.2191 & 325.7668 & 369.0693 \\
\hline & $\hat{\alpha}$ & \begin{tabular}{|l|l|}
0.025798 \\
\end{tabular} & \begin{tabular}{|l|}
0.036827 \\
\end{tabular} & 0.05373844 & 1.331674 & 1.0511818 & \begin{tabular}{|l|}
4.845107 \\
\end{tabular} \\
\hline \multirow{2}{*}{ MFD } & $\hat{\beta}$ & -2.746046 & -3.022394 & -3.55584431 & -7.889381 & -7.8376355 & -12.11829 \\
\hline & $\hat{\lambda}$ & 1.7597250 & 0.773008 & 0.39368449 & 0.256016 & 0.1480056 & \begin{tabular}{|l|l|}
0.100307 \\
\end{tabular} \\
\hline $\mathrm{RD}$ & $\hat{\sigma}$ & 420.0692 & 3313.579 & 1140.704 & 338.1586 & 432.1877 & 419.0887 \\
\hline
\end{tabular}

Table 3. Estimates of the parameters of statistical distribution for LNN.

\section{Concluding remarks}

This present study investigate implementation of a numerical solution of bioconvective nanofluid flow. The boundary layer flow (BLF) towards a vertical exponentially stretching plate with combination of heat and mass transfer rate in tangent hyperbolic nanofluid containing microorganisms. We have introduced zero mass flux condition to achieve physically realistic outcomes. Analysis is conducted with magnetic field phenomenon. By using similarity variables, the partial differential equation which governs the said model was converted into a nonlinear ordinary differential equation, and numerical results are achieved by applying the shooting technique. Bioconvective nanoliquid stream towards an expending surface and impacts of parameters $\mathrm{Nr}, \mathrm{Rb}, \mathrm{Lb}, \mathrm{Pe}, \mathrm{Nt}$ 


\begin{tabular}{|c|c|c|c|c|c|}
\hline & & \multicolumn{4}{|l|}{ LDMM } \\
\hline & & \multicolumn{4}{|l|}{$L b$} \\
\hline & & 1 & 2 & 3 & 4 \\
\hline \multirow{3}{*}{ F-D } & $\hat{\alpha}$ & 3.875291 & 4.192726 & 4.498246 & 4.795930 \\
\hline & $\hat{\beta}$ & 0.981468 & 1.053006 & 1.122236 & 1.188403 \\
\hline & $\hat{\eta}_{1}$ & 1.890055 & 2.260506 & 2.702876 & 3.220559 \\
\hline \multirow{3}{*}{ AGT-II } & $\hat{\delta}_{1}$ & 1.890055 & 2.260506 & 2.702876 & 3.220559 \\
\hline & $\hat{\eta}_{2}$ & 0.981294 & 1.052955 & 1.122235 & 1.188242 \\
\hline & $\hat{\delta}_{2}$ & 0.981294 & 1.052955 & 1.122225 & 1.188242 \\
\hline \multirow{2}{*}{ GT-II } & $\hat{\eta}$ & 0.981294 & 1.052957 & 1.122196 & 1.188337 \\
\hline & $\hat{\delta}$ & 3.780108 & 4.521043 & 5.406437 & 6.443316 \\
\hline \multirow{3}{*}{ Weibull } & $\hat{\lambda}$ & 1.663800 & 1.725847 & 1.786556 & 1.845495 \\
\hline & $\hat{\beta}$ & 9.108319 & 9.423109 & 9.734224 & 10.03794 \\
\hline & $\hat{\alpha}$ & 58.31327 & 79.70721 & 73.15271 & 89.53789 \\
\hline \multirow{2}{*}{ MFD } & $\hat{\beta}$ & 0.336251 & 0.332175 & 0.358084 & 0.359264 \\
\hline & $\hat{\lambda}$ & 0.161677 & 0.165079 & 0.162227 & 0.163958 \\
\hline $\mathrm{RD}$ & $\hat{\sigma}$ & 6.710372 & 6.880247 & 7.050128 & 7.217048 \\
\hline
\end{tabular}

Table 4. Estimates of the parameters of statistical distribution for LDMM.

\begin{tabular}{|l|l|l|l|l|l|l|l|}
\hline & \multicolumn{6}{|l|}{ SFC } \\
\hline & & \multicolumn{5}{|l}{$\boldsymbol{N}$} \\
\hline & & $\mathbf{0}$ & $\mathbf{1}$ & $\mathbf{2}$ & $\mathbf{3}$ & $\mathbf{4}$ & $\mathbf{5}$ \\
\hline \multirow{2}{*}{ F-D } & AIC & 43.71452 & 42.19349 & 40.68414 & 39.11847 & 37.4521 & 35.64968 \\
\cline { 2 - 8 } & BIC & 45.80357 & 44.28254 & 42.77318 & 41.20752 & 39.54114 & 37.73873 \\
\hline \multirow{2}{*}{ AGT-II } & AIC & 47.71452 & 46.1758 & 44.68414 & 43.11883 & 41.45449 & 39.64996 \\
\cline { 2 - 8 } & BIC & 51.89261 & 50.35389 & 48.86223 & 47.29692 & 45.63258 & 43.82805 \\
\hline \multirow{2}{*}{ GT-II } & AIC & 43.71452 & 42.19349 & 40.68416 & 39.12911 & 38.76079 & 40.4071 \\
\cline { 2 - 8 } & BIC & 45.80357 & 44.28254 & 42.7732 & 41.21815 & 40.84983 & 42.49614 \\
\hline \multirow{2}{*}{ Weibull } & AIC & $\mathbf{3 7 . 5 9 6 4 4}$ & $\mathbf{3 6 . 6 9 4 4 7}$ & $\mathbf{3 5 . 6 2 0 4 6}$ & $\mathbf{3 4 . 3 6 3 4 8}$ & $\mathbf{3 2 . 9 1 1 1 4}$ & $\mathbf{3 1 . 2 4 6 4 4}$ \\
\cline { 2 - 8 } & BIC & $\mathbf{3 9 . 6 8 5 4 8}$ & $\mathbf{3 8 . 7 8 3 5 1}$ & $\mathbf{3 7 . 7 0 9 5 1}$ & $\mathbf{3 6 . 4 5 2 5 2}$ & $\mathbf{3 5 . 0 0 0 1 9}$ & $\mathbf{3 3 . 3 3 5 4 8}$ \\
\hline \multirow{2}{*}{ MFD } & AIC & 42.44299 & 40.75795 & 39.58348 & 38.25849 & 36.76681 & 35.09028 \\
\cline { 2 - 8 } & BIC & 45.57655 & 43.89152 & 42.71705 & 41.39206 & 39.90037 & 38.22384 \\
\hline \multirow{2}{*}{ RD } & AIC & 47.46089 & 50.88832 & 54.10217 & 57.07796 & 59.81554 & 62.32395 \\
\cline { 2 - 8 } & BIC & 48.50541 & 51.93284 & 55.14669 & 58.12248 & 60.86006 & 63.36847 \\
\hline
\end{tabular}

Table 5. Akaike information criteria (AIC) and (BIC) for SFC.

\begin{tabular}{|c|c|c|c|c|c|c|c|}
\hline & & \multicolumn{6}{|l|}{ LNN } \\
\hline & & \multicolumn{6}{|l|}{$N t$} \\
\hline & & 6 & 7 & 8 & 9 & 10 & 11 \\
\hline \multirow{2}{*}{ F-D } & AIC & 39.59408 & 65.19384 & 86.95979 & 105.8262 & 122.4597 & 137.3348 \\
\hline & BIC & 41.01018 & 66.60994 & 88.37589 & 107.2423 & 123.8758 & 138.7509 \\
\hline \multirow{2}{*}{ AGT-II } & AIC & 81.71779 & 122.5622 & 154.628 & 182.0513 & 210.2601 & 223.8249 \\
\hline & BIC & 84.54999 & 125.3944 & 157.4602 & 184.8835 & 213.0923 & 226.6571 \\
\hline \multirow{2}{*}{ GT-II } & AIC & 80.71941 & 122.2521 & 154.9725 & 185.4515 & 212.4454 & 235.4214 \\
\hline & BIC & 82.13551 & 123.6682 & 156.3886 & 186.8676 & 213.8615 & 236.8375 \\
\hline \multirow{2}{*}{ Weibull } & AIC & 74.96524 & 100.7408 & 122.6578 & 141.6394 & 158.4089 & 222.6907 \\
\hline & BIC & 76.38134 & 102.1569 & 124.0739 & 143.0555 & 159.8250 & 224.1068 \\
\hline \multirow{2}{*}{ MFD } & AIC & 51.74538 & 75.66230 & 95.93718 & 114.3271 & 129.8048 & 144.3277 \\
\hline & BIC & 53.86953 & 77.78645 & 98.06133 & 116.4513 & 131.9289 & 146.4519 \\
\hline \multirow{2}{*}{$\mathrm{RD}$} & AIC & 296.6721 & 389.9711 & 298.727 & 204.4000 & 202.0818 & 202.9555 \\
\hline & BIC & 297.3802 & 390.6792 & 299.435 & 205.1081 & 202.7899 & 203.6636 \\
\hline
\end{tabular}

Table 6. Akaike information criteria (AIC) and (BIC) for the LNN. 


\begin{tabular}{|l|l|l|l|l|l|}
\hline & \multicolumn{4}{|l|}{ LDMM } \\
\hline & & \multicolumn{4}{l}{$\boldsymbol{l} \boldsymbol{b}$} \\
\hline & & $\mathbf{1}$ & $\mathbf{2}$ & $\mathbf{3}$ & $\mathbf{4}$ \\
\hline \multirow{2}{*}{ F-D } & AIC & 103.3815 & 102.9313 & 102.5711 & 102.2829 \\
\cline { 2 - 6 } & BIC & 104.7976 & 104.3474 & 103.9872 & 103.6990 \\
\hline \multirow{2}{*}{ AGT-II } & AIC & 107.3815 & 106.9313 & 106.5711 & 106.2829 \\
\cline { 2 - 6 } & BIC & 110.2137 & 109.7635 & 109.4033 & 109.1151 \\
\hline \multirow{2}{*}{ GT-II } & AIC & 103.3815 & 102.9313 & 102.5711 & 102.2829 \\
\cline { 2 - 6 } & BIC & 104.7976 & 104.3474 & 103.9872 & 103.699 \\
\hline \multirow{2}{*}{ Weibull } & AIC & $\mathbf{9 2 . 5 7 5 6 1}$ & $\mathbf{9 2 . 8 4 6 5}$ & $\mathbf{9 3 . 0 9 9 9}$ & $\mathbf{9 3 . 3 3 2 7 4}$ \\
\cline { 2 - 6 } & BIC & $\mathbf{9 3 . 9 9 1 7 1}$ & $\mathbf{9 4 . 2 6 2 6}$ & $\mathbf{9 4 . 5 1 6}$ & $\mathbf{9 4 . 7 4 8 8 4}$ \\
\hline \multirow{2}{*}{ MFD } & AIC & 95.8456 & 96.16195 & 96.54202 & 96.80884 \\
\cline { 2 - 6 } & BIC & 97.96975 & 98.2861 & 98.66617 & 98.93299 \\
\hline \multirow{2}{*}{ RD } & AIC & 91.35215 & 91.34132 & 91.3873 & 91.47751 \\
\cline { 2 - 6 } & BIC & 92.0602 & 92.04937 & 92.09535 & 92.18556 \\
\hline
\end{tabular}

Table 7. Akaike information criteria (AIC) and (BIC) for LDMM.

Skin Friction Coefficient $\mathrm{Nr}=0$

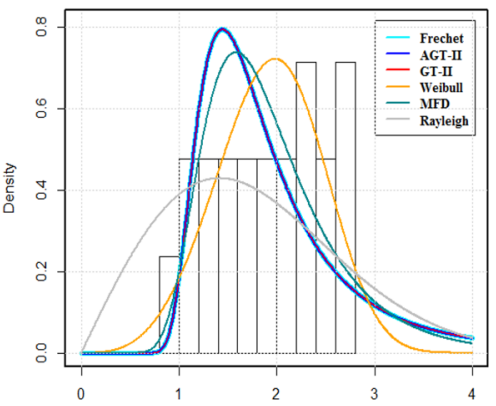

Skin Friction Coefficient $\mathrm{Nr}=3$

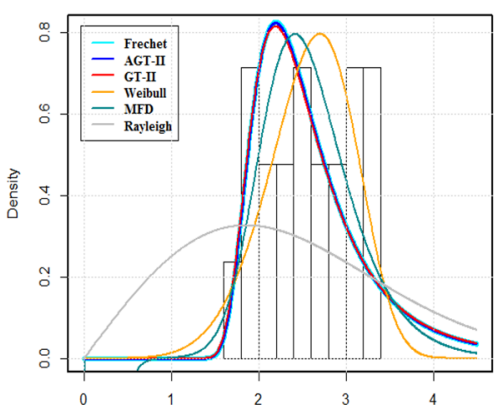

Skin Friction Coefficient

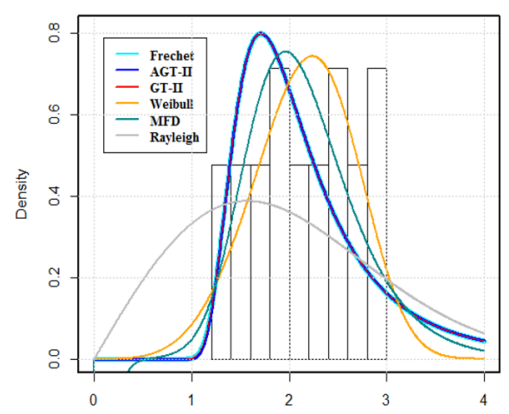

Skin Friction Coefficient $\mathrm{Nr}=4$

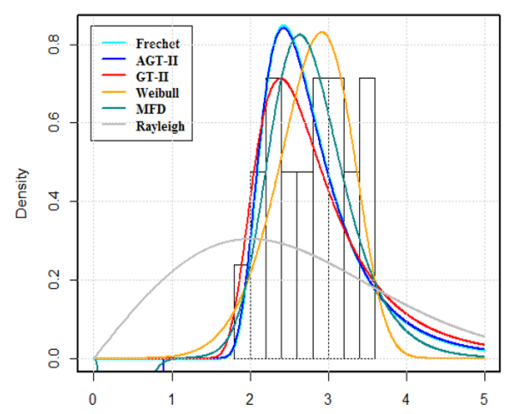

Skin Friction Coefficient $\mathrm{Nr}=2$

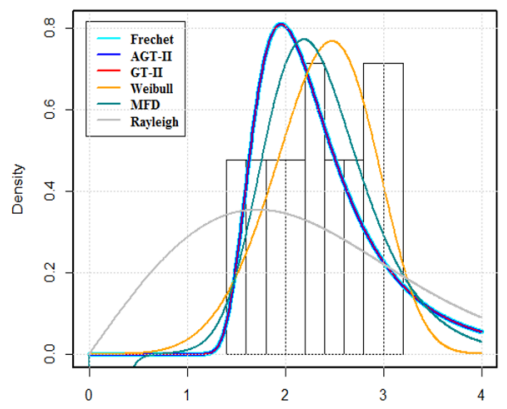

Skin Friction Coefficient $\mathrm{Nr}=5$

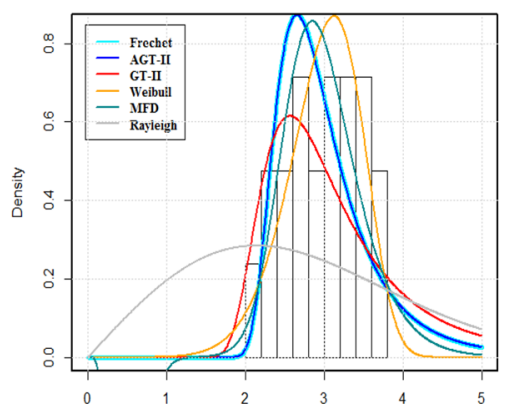

Figure 4. The estimated densities for the SFC.

and $\mathrm{Nb}$ is analyzed and studied. From this study, we obtain a unique solution for expanding surface. It is noted that, as $\mathrm{Nr}$ and $\mathrm{Rb}$ increases, the skin friction coefficient rises. The rate of mass transfer is increased by increasing $L b$ and $P e$. Furthermore, for least values of $N b$ and all the values of $\mathrm{Nt}$ under consideration the heat transfer rate upsurges. The data of SFC, LNN, and LDMM have been tested utilizing various statistical models, and it is noted that data sets for SFC and LDMM fit the Weibull model for different values of $N r$ and $L b$ respectively. On the other hand, Frechet distribution fits well for LNN data set for various values of $N t$. 

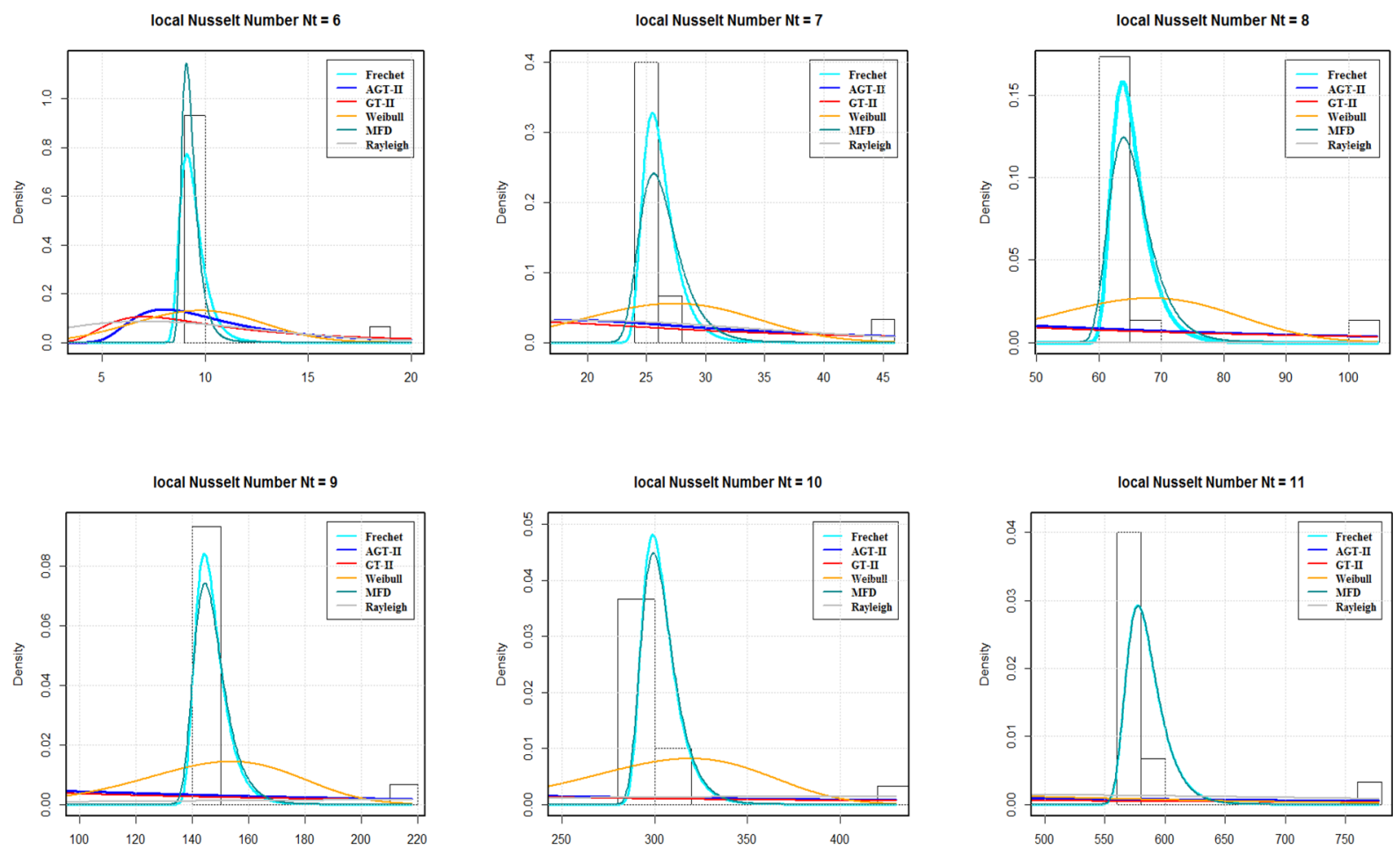

Figure 5. The estimated densities for the LNN. 
local density of motile microorganism $L b=1$

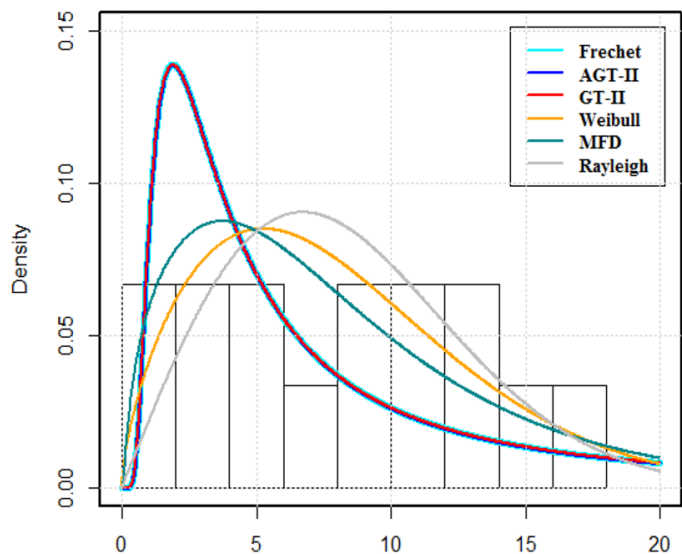

local density of motile microorganism $L b=3$

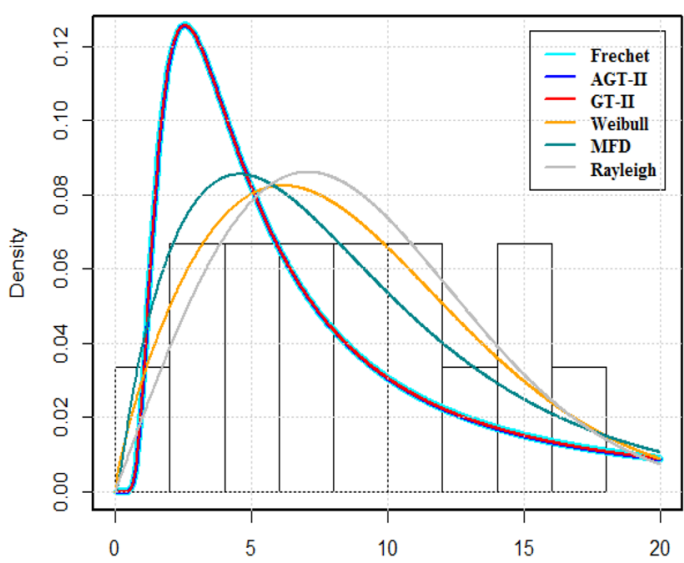

local density of motile microorganism $L b=2$

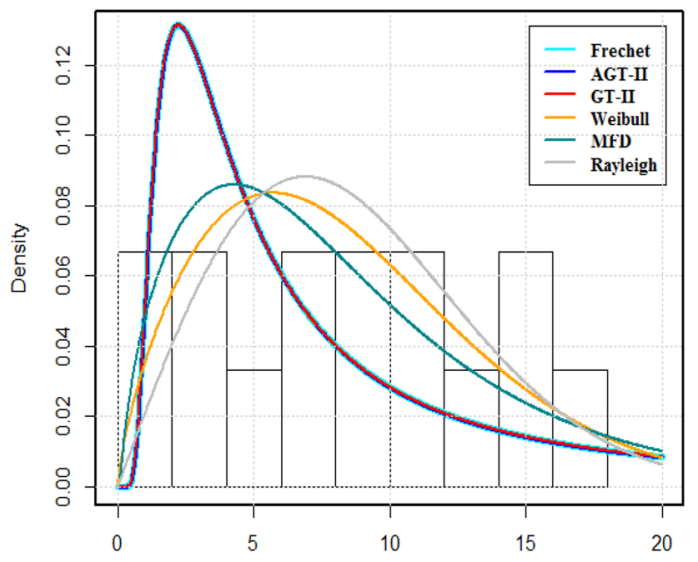

local density of motile microorganism Lb $=4$

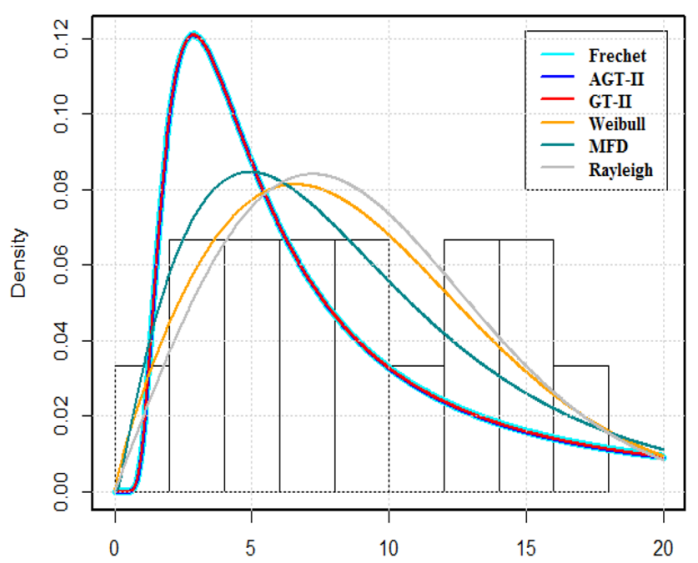

Figure 6. The estimated densities for the LDMM.

Received: 18 May 2021; Accepted: 22 June 2021

Published online: 06 July 2021

\section{References}

1. Crane, L. J. Flow past a stretching plate. Z. Angew. Math. Phys. 21, 645-647 (1970).

2. Gupta, P. S. \& Gupta, A. S. Heat and mass transfer of a continuous stretching surface with suction or blowing. Can. J. Chem. Eng. 55, 74-76 (1977).

3. Hayat, T., Shaheen, U., Shafiq, A., Alsaedi, A. \& Asghar, S. Marangoni mixed convection flow with Joule heating and nonlinear radiation. AIP Adv. 5, 077140 (2015).

4. Rasool, G., Shafiq, A., Khalique, C. M. \& Zhang, T. Magnetohydrodynamic Darcy-Forchheimer nanofluid flow over a nonlinear stretching sheet. Phys. Scripta 94(10), 105221 (2019).

5. Hayat, T., Shafiq, A., Alsaedi, A. \& Asghar, S. Effect of inclined magnetic field in flow of third grade fluid with variable thermal conductivity. AIP Adv. 5, 087108 (2015).

6. Rasool, G., Chamkha, A. J., Muhammad, T., Shafiq, A. \& Khan, I. Darcy-Forchheimer relation in Casson type MHD nanofluid flow over non-linear stretching surface. Propuls. Power Res. 9(2), 159-68 (2020).

7. Shafiq, A. \& Khalique, C. M. Lie group analysis of upper convected Maxwell fluid flow along stretching surface. Alex. Eng. J. 20, 20 (2020).

8. Hayat, T., Shafiq, A. \& Alsaedi, A. Effect of Joule heating and thermal radiation in flow of third-grade fluid over radiative surface. PLoS One 9(1), e83153 (2014).

9. Makinde, O. D. \& Aziz, A. Boundary layer flow of a nanofluid past a stretching sheet with a convective boundary condition. Int. J. Therm. Sci. 50, 1326-32 (2012).

10. Turkyilmazoglu, M. \& Pop, I. Heat and mass transfer of unsteady natural convection flow of some nanofluids past a vertical infinite flat plate with radiation effect. Int. J. Heat Mass Transf. 59, 167-171 (2013).

11. Mustafa, M., Hayat, T. \& Alsaedi, A. Unsteady boundary layer flow of nanofluid past an impulsively stretching sheet. J. Mech. 29, 423-432 (2013).

12. Ashorynejad, H. R., Sheikholeslami, M., Pop, I. \& Ganji, D. D. Nanofluid flow and heat transfer due to a stretching cylinder in the presence of magnetic field. Heat Mass Transf. 49, 427-436 (2013). 
13. Murthy, P. V. S. N., Ram Reddy, Ch., Chamkha, A. J. \& Rashad, A. M. Magnetic effect on thermally stratified nanofluid saturated non-Darcy porous medium under convective boundary condition. Int. Commun. Heat Mass Transf. 47, 41-48 (2013).

14. Rashidi, M. M., Abelman, S. \& Mehr, N. F. Entropy generation in steady MHD flow due to a rotating disk in a nanofluid. Int. J. Heat Mass Transf. 62, 515-525 (2013).

15. Jedi, A. et al. Statistical modeling for nanofluid flow: A stretching sheet with thermophysical property data. Colloids Interfaces 4, 3 (2020).

16. Chu, Y. M. et al. Examining rheological behavior of MWCNT-TiO2/5W40 hybrid nanofluid based on experiments and RSM/ANN modeling. J. Mol. Liq. 333, 115969 (2021).

17. Ali, V. et al. Navigating the effect of tungsten oxide nano-powder on ethylene glycol surface tension by artificial neural network and response surface methodology. Powder Technol. 386, 483-490 (2021).

18. Kuznetsov, A. V. Nanofluid bioconvection in water-based suspensions containing nanoparticles and oxytactic microorganisms: Oscillatory instability. Nanosc. Res. Lett. 6(100), 1-13 (2011).

19. Aziz, A., Khan, W. A. \& Pop, I. Free convection boundary layer flow past a horizontal flat plate embedded in porous medium filled by nanofluid containing gyrotactic microorganisms. Int. J. Thermal Sci. 56, 48-57 (2012).

20. Tham, L., Nazar, R. \& Pop, I. Mixed convection flow over a solid sphere embedded in a porous medium filled by a nanofluid containing gyrotactic microorganisms Int. J. Heat Mass Transf. 62, 647-660 (2013).

21. Ibrahim, M. Numerical analysis of time-dependent flow of viscous fluid due to a stretchable rotating disk with heat and mass transfer. Results Phys. 18, 103242 (2020).

22. Mutuku, W. N. \& Oluwole, D. K. Hydromagnetic bioconvection of nanofluid over a permeable vertical plate due to gyrotactic microorganisms. Comput. Fluids 95, 88-97 (2014).

23. Khan, W. A. \& Makinde, O. D. MHD nanofluid bioconvection due to gyrotactic microorganisms over a convectively heat stretching sheet. Int. J. Thermal Sci. 81, 118-124 (2014).

24. Khan, W. A., Uddin, M. J. \& Ismail, A. I. Free convection of nonNewtonian nanofluids in porous media with gyrotactic microorganisms. Transp. Porous Med. 97, 241-252 (2013).

25. Naseem, F., Shafiq, A., Zhao, L. \& Naseem, A. MHD biconvective flow of Powell Eyring nanofluid over stretched surface. Aip Adv. 7(6), 065013 (2017).

26. Shafiq, A., Sindhu, T. N. \& Khalique, C. M. Numerical investigation and sensitivity analysis on bioconvective tangent hyperbolic nanofluid flow towards stretching surface by response surface methodology. Alex. Eng. J.https://doi.org/10.1016/j.aej.2020.08.007 (2020).

27. Shafiq, A., Sindhu, T. N. \& Al-Mdallal, Q. M. A sensitivity study on carbon nanotubes significance in Darcy-Forchheimer flow towards a rotating disk by response surface methodology. Sci. Rep. 11(1), 1-26 (2021).

28. Shafiq, A., Hammouch, Z., Sindhu, T. N. \& Baleanu, D. Statistical approach of mixed convective flow of third-grade fluid towards an exponentially stretching surface with convective boundary condition. In Special Functions and Analysis of Differential Equations 307-319 (Chapman and Hall, 2020).

29. Shafiq, A., Rasool, G., Khalique, C. M. \& Aslam, S. Second grade bioconvective nanofluid flow with buoyancy effect and chemical reaction. Symmetry 12(4), $621(2020)$.

30. Shafiq, A., Hammouch, Z. \& Oztop, H. F. Radiative MHD flow of third-grade fluid towards a stretched cylinder. In International Conference on Computational Mathematics and Engineering Sciences 166-185 (Springer, 2019).

31. Mutuku, W. N. \& Makinde, O. D. Hydromagnetic bioconvection of nanofluid over a permeable vertical plate due to gyrotactic microorganisms. Comput. Fluids 95, 88-97 (2014).

32. Shafiq, A., Hammouch, Z. \& Sindhu, T. N. Bioconvective MHD flow of tangent hyperbolic nanofluid with newtonian heating. Int. J. Mech. Sci. 1(133), 759-66 (2017).

33. Kuznetsov, A. V. Bio-thermal convection induced by two different species of microorganisms. Int. Commun. Heat Mass Transf. 38, 548-553 (2011).

34. Acharya, N., Das, K. \& Kundu, P. K. Framing the effects of solar radiation on magneto-hydrodynamics bioconvection nanofluid flow in presence of gyrotactic microorganisms. J. Mol. Liq. 222, 28-37 (2016).

35. Akaike, H., Petrov, B. N., \& Csaki, F. Second international symposium on information theory (1973).

\title{
Author contributions
}

All authors have equal contribution.

\section{Competing intrests}

The authors declare no competing interests.

\section{Additional information}

Correspondence and requests for materials should be addressed to Q.M.A.-M.

Reprints and permissions information is available at www.nature.com/reprints.

Publisher's note Springer Nature remains neutral with regard to jurisdictional claims in published maps and institutional affiliations.

\begin{abstract}
Open Access This article is licensed under a Creative Commons Attribution 4.0 International License, which permits use, sharing, adaptation, distribution and reproduction in any medium or format, as long as you give appropriate credit to the original author(s) and the source, provide a link to the Creative Commons licence, and indicate if changes were made. The images or other third party material in this article are included in the article's Creative Commons licence, unless indicated otherwise in a credit line to the material. If material is not included in the article's Creative Commons licence and your intended use is not permitted by statutory regulation or exceeds the permitted use, you will need to obtain permission directly from the copyright holder. To view a copy of this licence, visit http://creativecommons.org/licenses/by/4.0/.
\end{abstract}

(C) The Author(s) 2021 